\title{
Psychological Factors Influencing Exercise Adherence among Young Adult Females in Pune City
}

\author{
By Ms. Ankita Dhone and Dr. M. R. Gaikwad
}

\begin{abstract}
The purpose of this review was to analyse empirical studies on Exercise adherence over the past 20 years and then propose recommendations for future research. A systematic process was used to search the literature for this review. Considering the present scenario and the area of interest the research topic is chosen "Psychological factors influencing exercise adherence among young adult females in Pune City" while going through the literature of related studies, the following reviews were collected.
\end{abstract}

\section{Body}

Dr Sudhinder Chowhan, Dr Srirang Jha and Mrs Vandana Malviya in their study "Anxiety in Working Women in Delhi: A Survey" concluded that that women do experience a higher level of stress as compared to men. The study looks into the stress levels of women working in varied organizations in Delhi. It is not only that state anxiety is higher among working women as compared to working women in Delhi but the trait anxiety is also higher among women. For study they used these tools A structured questionnaire- studying 5 dimensions like Maturity, Tension, Self Control, Guilt Proneness, and Suspiciousness was used. Their sample size was 100 where 50 were males and 50 were females. Whereas, Heather A. Kesselring worked on Title

"Experiences of women who are classified as maintainers and transformers for exercise"Ten women were interviewed individually in an attempt to discover how rearing or past experiences with exercise related to stage classification. The concepts that were examined through the interview process included family values and perceptions of exercise, periods of inactivity and the reasons for them, and the role of intrinsic motivation, enjoyment, and ritual in exercise adherence. The results provide evidence that successful exercisers do use the "helping relationships". What we do not know about maintainers and transformers is whether they began the change process with intrinsic goals and likewise, whether they always enjoyed their activity. In addition, there is no indication from any of the research whether personal upbringing impacts transformation or whether reutilization is an issue in transformation. Further, we do not know whether the under-use of "helping relationships" and "environmental control" impacts transformation. Further, we do not know whether the underuse of "helping relationships" and "environmental control" impacts transformation, likewise in this study certain recommendations were made which their study could not find out. In another literature "Explaining long-term exercise adherence in women who complete a structured exercise program". By Huberty JL, Ransdell

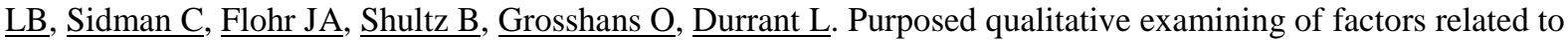
physical activity adherence to understand why women continue to participate in long-term exercise after completing a structured exercise program. Data were collected from focus groups, interviews, and e-mails, and analysis used grounded theory. The central category related to physical activity adherence was self-worth. Motivation, activity enjoyment, priorities, body image, ability to access support, and self-regulation skills had an impact on the self-worth of non-adherers and adherers. Women must value themselves enough to continue to participate in physical activity once they start. Exercise and fitness professionals are encouraged to use strategies to increase self-worth and long-term adherence to physical activity. Some recommended strategies include (a) increasing motivation and enjoyment relative to activity, (b) making activity a high priority in a woman's life, (c) improving or deemphasizing body image, (d) increasing a woman's ability to access support, and (e) facilitating the use of self-regulation strategies. This study is the first to examine qualitative perspectives of exercise adherence among women who completed a structured exercise program. Several concepts related to adherence presented in the quantitative literature are confirmed and enhanced in this study. This study states different ways to improve exercise adherence among women, but from my point of view this is not enough, some acute investigation and understanding is required. Further related study done

In 2005 by White JL, Ransdell LB, Vener J, Flohr JA on "Factors related to physical activity adherence in women: review and suggestions for future research". According to them approximately 50 percent of individuals who start an exercise program withdraw within 6 months. Thus, many individuals withdraw before health benefits have been realized. This is a disconcerting statistic considering the well known benefits of physical activity for decreasing risk of hypo kinetic diseases and improving quality of life. The literature has suggested a plethora of factors to increase the number of individuals who initiate a physical activity program. However, little is known about the factors that keep women exercising-otherwise known as exercise adherence. The purpose of this paper was to: (a) systematically review the quantitative literature to discern the major factors 
contributing to adherence to physical activity in women and men and make recommendations for specific gender-based considerations that are important when designing PA interventions for women, and (b) suggest areas of future research related to increasing adherence to physical activity in women. Key factors reviewed in this paper may be useful in developing efficacious physical activity programs for women. In this paper the purpose was not perfectly clarified. One more similar literature found on "Maintaining regular physical activity in women: evidence to date.” By Speck BJ ${ }^{1}$, Harrell JS. In 2003 Sep-Oct which statesPhysical activity is well established as a primary health promotion and cardiovascular disease prevention behavior, yet over $60 \%$ of the population does not participate in regular physical activity. Maintenance of regular physical activity is a complex behavior influenced by variables from several domains. This review includes studies of the relationship between physical activity in women and psychological, social environmental, demographic, physiologic, health status, and physical activity variables. A key issue in physical activity research is development of interventions for maintaining a physically active lifestyle. Interventions for maintenance of regular physical activity that have a strong theoretical basis will be beneficial to women in primary care settings, occupational settings, wellness centers as well as public health departments and other organizations that address the needs of women. This theory based study suggests development of interventions for exercise adherence in women which can differ from environment to environment. The further basic study done in 2004 Mar on"Physical activity and exercise in women's health.” By Belza B ${ }^{1}$, Warms C. states Regular physical activity provides health benefits, including the reduction in risks of coronary heart disease, hypertension, type 2 diabetes mellitus, obesity, colon cancer, and premature mortality. Despite this information, most women are physically inactive. Research findings shed light on the gender differences in physiological responses to physical activity. Patterns and predictors of physical activity vary significantly by gender. Further study is needed of the benefits, barriers, and personally meaningful outcomes of physical activity for women, specifically including the frequently unspoken correlates of urinary incontinence, depression and mood disorders, and obesity.

\section{Overall Conclusion}

Given the evidence that forms the basis for the present review of literature on health benefits from physical activity adherence in women in the prevention and management of disease, and the risks of being physically inactive, it appears that the current burden for health care systems could be eased by developing a more physically active women population. Although all people can achieve a variety of benefits from participating in regular physical activity and adhering to it, considerable improvements in population health can occur by focusing health promotion efforts on those sub-populations least likely to achieve sufficient physical activity, such as women.

\section{References}

[1]. al., H. J. (2008, sept.). explaining long term exercise adherence in women who complete a structured exercise program. Retrieved from https://www.ncbi.nlm.nih.gov/pubmed/18816949

[2]. Belza B., W. C. (2004, march). physical activity and exercise in womens health. Retrieved from https://www.ncbi.nlm.nih.gov/pubmed/15062735

[3]. Kesselring, A. (2002, january/ february). journal of rehabilitation, research and development. Retrieved 2002

[4]. Ransdell LB, e. a. (n.d.). factors related to physical activity adherence in women. Retrieved from https://www.ncbi.nlm.nih.gov/pubmed/16260417

[5]. Speck BJ, H. J. (2003, sept.-oct.). maintaining regular physical activity in women: evidence to date. Retrieved from https://www.ncbi.nlm.nih.gov/pubmed/14518604 